\title{
Retinoid metabolizing enzymes in development
}

\author{
Eric C. Swindell ${ }^{\mathrm{a}}$ and Gregor Eichele $\mathrm{a}^{\mathrm{a}, \mathrm{b}}$ \\ ${ }^{a}$ Verna and Marrs McLean Department of Biochemistry, Baylor College of Medicine, \\ One Baylor Plaza, Houston, TX 77030, USA \\ ${ }^{\mathrm{b}}$ Max-Planck Institute for Experimental Endocrinology, Feodor-Lynen-Strasse 7, \\ 30625 Hannover, Germany
}

Received 2 August 1998

Accepted 5 September 1998

\section{Introduction}

It has long been known that retinoids are necessary for normal embryogenesis. To understand the role of retinoids in this process, it is important to elucidate the retinoid signalling pathway. The retinoid signal transduction pathway consists of three major components: (i) the metabolism of biologically active retinoids, (ii) nuclear receptors which bind to and are activated by retinoids, and (iii) the genes whose expression is regulated by the receptor/ligand complex. The initiation of the retinoid signal transduction pathway critically depends of the presence of biologically active retinoids. The production and degradation of these biologically active retinoids will be the main focus this brief review. In addition, we will discuss the developmental aspects of retinoid metabolism.

Several retinoids including all-trans-retinoic acid, 9-cis-retinoic acid, 4-oxo-all-trans-retinoic acid, and 3,4-didehydro-all-trans-retinoic acid have been shown to be biologically active in the developing embryo [7-10,13,17,18,32,33,35]. The all-trans isomers of these biologically active retinoids bind to retinoic acid receptors (RARs) while 9-cis-retinoic acid binds to retinoid X receptors (RXRs) as well as to RARs. RARs and RXRs belong to the family of nuclear hormone receptors. These are multidomain transcription factors that bind to specific enhancer elements (retinoic acid response elements or RAREs) in a ligand dependent fashion [23,24]. Typically, RAR's and RXR's bind to enhancer elements as heterodimers. In the absence of ligand, a co-repressor is bound to this RXR/RAR heterodimer causing repression of transcription from the associated promoter. Upon binding of ligand, the co-repressor is released and transcription is activated through binding of a co-activator. Ultimately, co-activators and co-repressors affect the chromatin structure of the ligand-regulated gene [16,37]. 


\section{Retinoid metabolic pathway}

Retinol is converted to retinoic acid (RA) by two successive dehydrogenation reactions. A retinol dehydrogenase $(\mathrm{RoDH})$ converts retinol to retinal in the first step. This step is rate limiting and reversible [6]. In the second non-reversible step, a retinal dehydrogenase (RalDH) converts retinal to RA. Although these reactions have been known to occur for some time [11], the enzymes that carry out these reactions have only recently been characterized at a molecular level. To date, several RoDH and RalDH isozymes have been isolated $([12,27]$ for review).

The degradation of RA is thought to begin with the conversion of RA into more polar metabolites, such as 4-OH-RA and 4-oxo-RA. A novel cytochrome P450, P450 RAI (also known as CYP26), has recently been isolated that converts RA to 4-OH-RA, 4-oxo-RA and 18-OH-RA [15,30,38,39]. To date, P450 RAI is the only enzyme isolated that is capable of degrading RA. The above enzymes act in concert to control the homeostasis of RA in the embryo and ensure the proper regulation of retinoid target genes. Although not discussed here, cellular retinoid binding proteins are also thought to be important in the control of RA homeostasis ([18] for review).

\subsection{Conversion of retinol to retinal}

Several retinol dehydrogenases (RoDHs) have been isolated and characterized with respect to expression pattern in the developing embryo. A class I alcohol dehydrogenase (class I ADH) is expressed in kidney at embryonic day 9.5 (E 9.5), while a class III ADH has been isolated that is expressed throughout the embryo from stage E 6.5 to E 9.5 [2]. In addition, a class IV ADH has been isolated that shows expression restricted to the mesoderm of the primitive streak starting at $\mathrm{E} 7.5$ [1,2]. In older embryos, class IV ADH is expressed in the dorsal neural fold, somites, paraxial mesoderm, neural crest, the proximal portion of the forelimb bud, and in craniofacial primordia [2]. Recently, an enzyme has been identified in both mouse and human that specifically converts 9-cis-retinol into 9-cis-retinaldehyde, a precursor to 9-cis-RA [25,31].

\subsection{Conversion of retinal to retinoic acid}

Several retinaldehyde dehydrogenases (RalDHs) have been identified that can convert retinal to RA. Two of these, class I ALDH [1] and RALDH-2 [28] are expressed in the primitive streak mesoderm similar to the expression of class IV ADH. The expression patterns of these enzymes support the idea that the primitive streak region of the early embryo is a site of RA production and this locally generated RA may control the expression of RA-responsive homeobox (Hox) genes at this developmental stage. At later stages, class I ALDH is expressed in somites, paraxial mesoderm, dorsal retina and craniofacial mesenchyme [1] while RALDH-2 is expressed in somites, the optic vesicle, interdigital tissue of the limb, tooth buds, the inner ear and the pituitary gland. These later expression patterns are also similar to the expression of class IV ADH in later stage embryos [1,2].

\subsection{Conversion of retinoic acid to 4-OH-RA, 4-oxo-RA and 18-OH-RA}

The degradation of RA is thought to begin with the formation of polar intermediates such as 4-OH-RA and 4-oxo-RA [14]. A novel cytochrome P450 has been isolated, P450 ${ }^{\mathrm{RAI}}$, that is inducible by RA and can convert RA into 4-OH-RA, 18-OH-RA and 4-oxo-RA [30,38,39]. Fujii et al. [15] have also cloned a cytochrome P450 that they believe to be different from P450 $0^{\mathrm{RAI}}$ based on the metabolites produced. 
However, sequence comparison of the two proteins from mouse show that they are identical. Expression of $P 450^{R A I}$ has been described in the mouse by Fujii et al. [15]. At E 7, extraembryonic and embryonic endoderm and embryonic mesoderm express $P 450^{R A I}$. By E 7.25 transcripts are detected in the primitive streak region. By E 7.5, expression in the primitive streak region has greatly diminished, but $P 450^{R A I}$ transcripts are now found in the anterior part of the embryo in all three germ layers. By E 8.5, the gene is highly expressed in the tailbud mesoderm, the posterior neural plate, and at the anterior end of the embryo including the hindbrain, the foregut, and the first pharyngeal arch (a primordium of the vertebrate face). By E 9.5 to 10.5 transcripts are found at the dorsoventral boundary of the retina, the caudal most part of the embryo and in neural crest cells that contribute to the cranial ganglia. These cells are known to be sensitive to excess RA and one role of P450 ${ }^{\mathrm{RAI}}$ may be to keep RA levels low in these cells. P450 ${ }^{\mathrm{RAI}}$ is also found in the limb bud as well as in the interdigital space. Of note, treatment of embryos with RA resulted in an abolishment of the caudal expression domain of $\mathrm{P} 450^{\mathrm{RAI}}$ and at the same time, in an upregulation of expression of this gene in the cranial region. Thus in the embryo, this gene is differentially regulated by RA. It will be interesting to compare the expression patterns of the RA-producing enzymes (RoDHs and RalDHs) with that of P450 ${ }^{\mathrm{RAI}}$. The present data suggest that in the early embryo (E 7.5 and before) the two types of enzymes are co-expressed in the primitive streak region. This is somewhat of a paradox, since it would mean that locally produced RA is degraded at once. A future task will be to determine whether additional retinoid metabolism enzymes exist and where and when precisely these are expressed. Although the research on RA synthesis and degradation is still in its infancy, information about the concentrations of RA in tissue is a critical factor in determining receptor-mediated gene activation.

\section{Developmental consequences of retinoid metabolism}

Many genes involved in early brain formation and patterning have been shown to be responsive to RA [3-5,34]. In fact, several of these have been shown to contain RAREs that are essential for normal expression $[20,26]$. Ectopic RA application has been shown to cause a mirror-symmetrical digit duplication in the developing limb bud [36] and the blocking of the retinoid signalling pathway during early determination of the wing field has been shown to disrupt normal wing formation [21]. Proper development of organs such as the heart have been shown to be sensitive to RA [29] and vitamin A-deficient embryos show marked abnormalities in the heart and in other parts of the cardiovascular system [22]. RA has also been implicated in determination of the ventral retina [19]. Although detailed expression patterns of class I ALDH, RALDH-2 and P450 ${ }^{\mathrm{RAI}}$ have not been carried out in all of these tissues and organs, some of the RA metabolizing enzymes show interesting expression patterns in those tissues and organs known to depend on RA signal transduction. This suggests that these retinoid metabolizing enzymes play a major role in the proper formation and differentiation of these tissues and organs.

\section{References}

[1] H.L. Ang and G. Duester, Initiation of retinoid signaling in primitive streak mouse embryos: spatiotemporal expression patterns of receptors and metabolic enzymes for ligand synthesis, Developmental Dynamics 208 (1997), 536-543.

[2] H.L. Ang, T.F. Hayamizu, M. Zgombic-Knight and G. Duester, Retinoic acid synthesis in mouse embryos during gastrulation and craniofacial development linked to class IV alcohol dehydrogenase gene expression, Journal of Biological Chemistry 271 (1997), 9526-9534.

[3] V. Avantaggiato, D. Acampora, F. Tuorto and A. Simeone, Retinoic acid induces stage-specific repatterning of the rostral central nervous system, Developmental Biology 175 (1996), 347-357. 
[4] L. Bally-Cuif, M. Gulisano, V. Broccoli and E. Boncinelli, c-otx2 is expressed in two different phases of gastrulation and is sensitive to retinoic acid treatment in chick embryo, Mechanisms of Development 49 (1995), 49-63.

[5] R.A. Conlon, Retinoic acid and pattern formation in vertebrates, Trends in Genetics 11 (1995), 314-319.

[6] H. Chen, M.J. Namkung and M.R. Juchau, Biotransformation of all-trans-retinol and all-trans-retinal to all-trans-retinoic acid in rat conceptal homogenates, Biochem. Pharmacol. 50 (1995), 157-1264.

[7] Y. Chen, L. Huang and M. Solursh, A concentration gradient of retinoids in the early Xenopus laevis embryo, Developmental Biology 161 (1994), 70-76.

[8] Y.-P. Chen, L. Huang, A.F. Russo and M. Solursh, Retinoic acid is enriched in Hensen's node and is developmentally regulated in the early chicken embryo, Proc. Natl. Acad. Sci. USA 89 (1992), 10 056-10 059.

[9] P. Costaridis, C. Horton, J. Zeitlinger, N. Holder and M. Maden, Endogenous retinoids in the zebrafish embryo and adult, Developmental Dynanics 205 (1996), 41-51.

[10] J. Creech Kraft, T. Schuh, M. Juchau and D. Kimelman, The retinoid X receptor ligand, 9-cis-retinoic acid, is a potential regulator of early Xenopus development, Proc. Natl. Acad. Sci. USA 91 (1994), 3067-3071.

[11] J.E. Dowling and G. Wald, The biological function of vitamin A acid, Proc. Natl. Acad. Sci. USA 46 (1960), 587-608.

[12] G. Duester, Involvement of alcohol dehydrogenase, short-chain dehydrogenase/reductase, aldehyde dehydrogenase, and cytochrome P450 in the control of retinoid signaling by activation of retinoic acid synthesis, Biochemistry 35 (1996), $12221-12227$.

[13] A.J. Durston, J.P.M. Timmermans, W.J. Hage, H.F.J. Hendriks, N.J. de Vries, M. Heideveld and P.D. Nieuwkoop, Retinoic acid causes an anteroposterior transformation in the developing central nervous system, Nature 340 (1989), 140-144.

[14] C.A. Frolik, A.B. Roberts, T.E. Tavela, P.P. Roller, D.L. Newton and M.B. Sporn, Isolation and identification of 4-hydroxyand 4-oxoretinoic acid, Biochemistry 18 (1979), 2092-2097.

[15] H. Fujii, T. Sato, S. Kaneko, O. Gotoh, Y. Fulii-Kuriyama, K. Osawa, S. Kato and H. Hamada, Metabolic inactivation of retinoic acid by a novel P450 differentially expressed in developing mouse embryo, EMBO 16 (1997), 4163-4173.

[16] C.K. Glass, D.W. Rose and M.G. Rosenfeld, Nuclear receptor coactivators, Curr. Opin. Cell Biol. 9 (1997), $222-232$.

[17] B.L.M. Hogan, C. Thaller and G. Eichele, Evidence that Hensen's node is a site of retinoic acid synthesis, Nature 359 (1992), 237-241.

[18] C. Horton and M. Maden, Endogenous distribution of retinoids during normal development and teratogenesis in the mouse embryo, Developmental Dynanics 202 (1995), 312-323.

[19] G.A. Hyatt, E.A. Schmitt, N. Marsh-Armstrong, P. McCaffery, U.C. Drager and J.E. Dowling, Retinoic acid establishes ventral retinal characteristics, Development 122 (1996), 195-204.

[20] A.W. Langston, J.R. Thompson and L.J. Gudas, Retinoic acid-responsive enhancers located $3^{\prime}$ of the Hox A and Hox B gene clusters, Journal of Biological Chemistry 272 (1997), 2167-2175.

[21] H.-C. Lu, J.-P. Revelli, L. Goering, C. Thaller and G. Eichele, Retinoids signaling is required for the establishment of a ZPA and for the expression of Hoxb-8, a mediator of ZPA formation, Development 124 (1997), 1643-1651.

[22] M. Maden, E. Gale, I. Kostetskii and M. Zile, Vitamin A-deficient quail embryos have half a hindbrain and other neural defects, Current Biology 6 (1996), 417-426.

[23] D.J. Mangelsdorf and R.M. Evans, The RXR heterodimers and orphan receptors, Cell 83 (1995), 841-850.

[24] D.J. Mangelsdorf, K. Umesono and R.M. Evans, The retinoid receptors, in: The Retinoids, M.B. Sporn, A.B. Roberts and D.S. Goodman, eds, Ravern Press, Ltd., New York, 1994, pp. 319-349.

[25] J.R. Mertz, E. Shang, R. Piantedosi, S. Wei, D.J. Wolgemuth and W.S. Blaner, Identification and characterization of a steriospecific human enzyme that catalyzes 9-cis-retinol oxidation, Journal of Biological Chemistry 272 (1997), 11744 11749.

[26] H. Marshall, M. Studer, H. Popperl, S. Aparicio, A. Kuroiwa, S. Brenner and R. Krumlauf, A conserved retinoic acid response element required for early expression of the homeobox gene Hoxb-1, Nature 370 (1994), 567-571.

[27] J.L. Napoli, Retinoic acid biosynthesis and metabolism, FASEB 10 (1996), 993-1001.

[28] K. Niederreither, P. McCaffery, U.C. Dräger, P. Chambon and P. Dollé, Restricted expression and retinoic acid-induced downregulation of the retinaldehyde dehydrogenase type 2 (RALDH-2) gene during mouse development, Mechanisms of Development 62 (1997), 67-78.

[29] M.K. Osmond, A.J. Butler, F.C.T. Voon and R. Bellairs, The effects of retinoic acid on heart formation int he early chick embryo, Development 113 (1991), 1405-1417.

[30] W.J. Ray, G. Bain, M. Yao and D.I. Gottlieb, CYP26, a novel mammalian cytochrome P450, is induced by retinoic acid and defines a new family, Journal of Biological Chemistry 272 (1997), 18 702-18 708.

[31] A. Romert, P. Tuvendal, A. Simon, L. Dencker and U. Eriksson, The identification of a 9-cis retinol dehydrogenase in the mouse embryo reveals a pathway for synthesis of 9-cis retinoid acid, PNAS 95 (1998), 4404-4409.

[32] W.J.J. Scott, R. Walter, G. Tzimas, S.J. O, H. Nau and M.D. Collins, Endogenous status of retinoids and their cytosolic binding proteins in limb buds of chick vs mouse embryos, Developmental Biology 165 (1994), 397-409.

[33] T. Stratford, C. Horton and M. Maden, Retinoic acid is required for the formation of outgrowth in the chick limb bud, Current Biology 6 (1996), 1124-1133. 
[34] O. Sundin and G. Eichele, An early marker of axial pattern in the chick embryo and its respecification by retinoic acid, Development 114 (1992), 841-852.

[35] C. Thaller and G. Eichele, Identification and spatial distribution of retinoids in the developing chick limb bud, Nature $\mathbf{3 2 7}$ (1987), 625-628.

[36] C. Tickle, B. Alberts, L. Wolpert and J. Lee, Local application of retinoic acid to the limb bud mimics the action of the polarizing region, Nature 296 (1982), 564-566.

[37] J. Torchia, C. Glass and M.G. Rosenfeld, Co-activators and co-repressors in the integration of transcriptional responses, Curr. Opin. Cell Biology 10 (1998), 373-383.

[38] J.A. White, B. Beckett-Jones, Y.-D. Guo, F.J. Dilworth, J. Bonasoro, G. Jones and M. Petkovich, cDNA cloning of human retinoic acid-metabolizing enzyme (hP450RAI) identifies a novel family of cytochromes P450 (CYP26), Journal of Biological Chemistry 272 (1997), 18 538-18 541.

[39] J.A. White, Y.-D. Guo, K. Baetz, B. Beckett-Jones, J. Bonasoro, K.E. Hsu, F.J. Dilworth, G. Jones and M. Petkovich, Identification of the retinoic acid-inducible all-trans-retinoic acid 4-hydroxylase, Journal of Biological Chemistry 271 (1996), 29922-29927. 